\title{
Behcet's Disease: Presented with Genital Ulcer
}

\author{
Sayma Afroz ${ }^{1}$, Gulshan Ara $^{2}$ \\ Received: January 19, 2015 Accepted: July 10, 2015 \\ doi: http://dx.doi.org/10.3329/jemc.v5i3.24751
}

\begin{abstract}
Behcet's disease which can affect almost every organ system of the body and is diagnosed mainly clinically is a rare condition. The presence of certain clinical features, elimination of other possible causes of patient's symptoms and if possible proof of vasculitis by biopsy of an involved tissue supports a diagnosis. We report a young female presented with 7-day history of vulval ulcer and erythema nodosum. Diagnosis was made according to International Study Group criteria for Behcet's disease and histological findings. She was treated with corticosteroid, colchicine and dapsone which caused significant clinical improvement. We report this case to increase awareness among physicians on Behcet's disease to improve its management.
\end{abstract}

Key words: Behcet's disease; Genital ulcer; Vasculitis

J Enam Med Col 2015; 5(3): 175-178

\section{Introduction}

Behcet's disease ${ }^{1}$ or Behcet disease, sometimes called Behcet's syndrome, Morbus Behcet, BehcetAdamantiades syndrome ${ }^{2}$ or Silk Road disease, is a rare immune-mediated small-vessel systemic vasculitis. ${ }^{3}$ In 1937 it was named after the Turkish dermatologist Hulusi Behcet who first described the triple-symptom complex of recurrent oral aphthous ulcers, genital ulcers, and uveitis. As a systemic disease, it can also involve visceral organs and can be fatal due to ruptured vascular aneurysms or severe neurological complications. $^{4}$

Behcet's disease exists in many parts of the world with high incidences in the Middle East, Far East and Mediterranean regions and in an area of the ancient trading route known as 'Old Silk Road'.5 High prevalence $(420$ per 100,000$)$ has been reported in Turkey ${ }^{6}$ with lowest prevalence of 0.38 per 100000 being reported in the North America. ${ }^{7}$ The prevalence ranges from 2-30/100000 populations in Asian countries. ${ }^{8}$

Behcet's disease is believed to be caused by autoimmune response to an infectious or environmental insult in a genetically predisposed individual. HLA-B51 is the most strongly associated genetic risk factor. Behcet's disease typically presents in the third and fourth decade of life with no specific sex predilection. $5,7,9$

Nearly all patients present with some form of painful oral mucocutaneous ulcerations in the form of aphthous ulcers or non-scarring oral lesions. ${ }^{4}$ Painful genital ulcerations usually develop around the anus, vulva or scrotum and cause scarring in $75 \%$ of the patients. ${ }^{4}$ Additionally, patients may present with erythema nodosum, cutaneous pustular vasculitis and lesions similar to pyoderma gangrenosum. ${ }^{4}$ Inflammatory eye disease can develop early in the disease course and leads to permanent vision loss in $20 \%$ of cases. ${ }^{7,9}$ Gastrointestinal manifestations include abdominal pain, nausea, and diarrhoea with or without blood. ${ }^{4}$ Lung involvement is typically in the form of haemoptysis, pleuritis, cough, or fever. ${ }^{4}$ Arthralgia is seen in up to half of the patients and is usually due to non-erosive

1. Assistant Professor, Department of Obstetrics \& Gynaecology, Enam Medical College \& Hospital, Savar, Dhaka

2. Professor, Department of Obstetrics \& Gynaecology, Enam Medical College \& Hospital, Savar, Dhaka

Correspondence Sayma Afroz, Email: saymaafroz@yahoo.com 
poly- or oligoarthritis primarily of the large joints of the lower extremities. ${ }^{4}$ Neurological involvements range from aseptic meningitis to vascular thrombosis such as dural sinus thrombosis and organic brain syndrome manifesting with confusion, seizures, and memory loss. ${ }^{4}$ Pericarditis is a frequent cardiac manifestation. ${ }^{10}$ Chronic aortic regurgitation due to aortic root disease may also be seen. ${ }^{11}$

According to the International Study Group Guidelines for Behcet's Disease (ISGBD), for a patient to be diagnosed with Behçet's disease ${ }^{1}$, the patient must have oral (aphthous) ulcers (any shape, size or number at least 3 times in any 12 months period) along with 2 out of the following 4 "hallmark" symptoms:

1. Genital ulcers

2. Skin lesions (papulo-pustules, folliculitis, erythema nodosum, acne)

3. Eye inflammation (iritis, uveitis, retinal vasculitis, cells in the vitreous)

4. Pathergy reaction (appearance of papule $>2 \mathrm{~mm}$ diameter or sterile pustule $24-48 \mathrm{hrs}$ or more after an intradermal needle-prick). The pathergy test has a specificity of $95 \%$ to $100 \%$, but sensitivity is less. ${ }^{1}$

Histologic findings are not diagnostic but suggestive which reveals leucocytoclastic vasculitis with perivascular infiltration with neutrophils and lymphocytes. Other tests may be performed to rule out other diseases that may appear similar to Behcet's disease.

The ISGBD criteria created in 1990 have excellent specificity, but lack sensitivity. ${ }^{1}$ The International Criteria for Behcet's Disease (ICBD) was created in 2006, as replacement to ISG. For ISG oral aphthous ulcer is mandatory. For ICBD vascular lesions were added while oral aphthosis is no more mandatory. Vascular manifestation is defined as superficial phlebitis, deep vein thrombosis, large vein thrombosis, arterial thrombosis and aneurysm. Therefore ICBD use six items: oral ulcer, genital ulcer, skin lesions, eye lesions, vascular lesion and positive pathergy test. In the ICBD, genital aphthous lesions and eye lesions have more diagnostic value than the others. They get each 2 points. The other 4 items get one point each. A patient has to get 3 or more points to be diagnosed as having Behcet's disease. $^{11}$
The treatment plan for Behcet's disease varies depending on the severity of symptoms, the presence of complications, a person's age and medical history and other factors. Behcet's disease cannot be cured, but treatment can help to reduce symptoms and minimize the development of serious complications, such as meningitis, blindness and aneurysm of the lungs. Corticosteroids, immunosuppressant drugs, immunomodulators, tumor necrosis factor-inhibitors, and other symptomatic treatments are commonly used in the management. ${ }^{11}$

The European League Against Rheumatism (EULAR) recommendations for the management of Behcet's disease were developed in 2008 and guide the management of different aspects of Behcet's disease. ${ }^{12}$ For oral and genital ulcerations topical steroids or sucralfate solution is $1^{\text {st }}$ line therapy for mild lesions. For severe lesions, systemic corticosteroids, azathioprine, dapsone, interferon alpha, colchicine and thalidomide have demonstrated benefit. ${ }^{12}$ Behcet's disease usually has a benign course with lifelong remissions and recurrences.

\section{Case report}

A previously healthy 29-year-old lady, mother of two children, of middleclass family was admitted in Department of Obstetrics \& Gynaecology, Enam Medical College \& Hospital with the complaints of pain and ulceration on vulva for 7 days. She also complained of painful rashes on skin of upper and lower extremities for last 7 days. She gave history of taking injection benzathine penicillin, tablet azithromycin and acyclovir cream for last 5 days with no improvement. On inquiry she gave history of recurrent oral ulcer at least 4 to 5 times in last one year but she did not consult any physician for this. On general examination, all parameters were normal except erythema nodosum which are nodular erythematous tender rashes over skin of upper and lower limbs. On local examination of the vulva there was extensive confluent ulceration with necrotic base with foul smelling pus on ulcers on both sides of labia majora and minora (Fig 1). On palpation the ulcer was tender, deep with irregular margin measuring about $6 \mathrm{~cm} \times 4 \mathrm{~cm}$ on right and $5 \mathrm{~cm} \times 3 \mathrm{~cm}$ on left side. On per vaginal examination, vagina and cervix were healthy, uterus was anteverted with normal size and all fornices were free. There was no inguinal lymphadenopathy. On investigation, complete blood 
count showed neutrophilic leucocytosis with raised ESR, urine routine examination showed 18-20 pus cells, random blood sugar $6 \mathrm{mmol} / \mathrm{L}$, VDRL nonreactive, serological test for HIV 1 and 2 negative, serum creatinine $68 \mu \mathrm{mol} / \mathrm{L}$, pathergy test negative, histopathology report of biopsy from ulcer showed angiocentric mononuclear cells and neutrophilic infiltrate with few extravasation of RBCs which are compatible with Behcet's disease. Diagnosis of Behcet's disease was made according to ISGBD criteria based on the presence of recurrent oral ulcer together with vulval ulcer, skin rash and erythema nodosum. Histopathology of punch biopsy from ulcer margin was also suggestive of Behcet's disease.

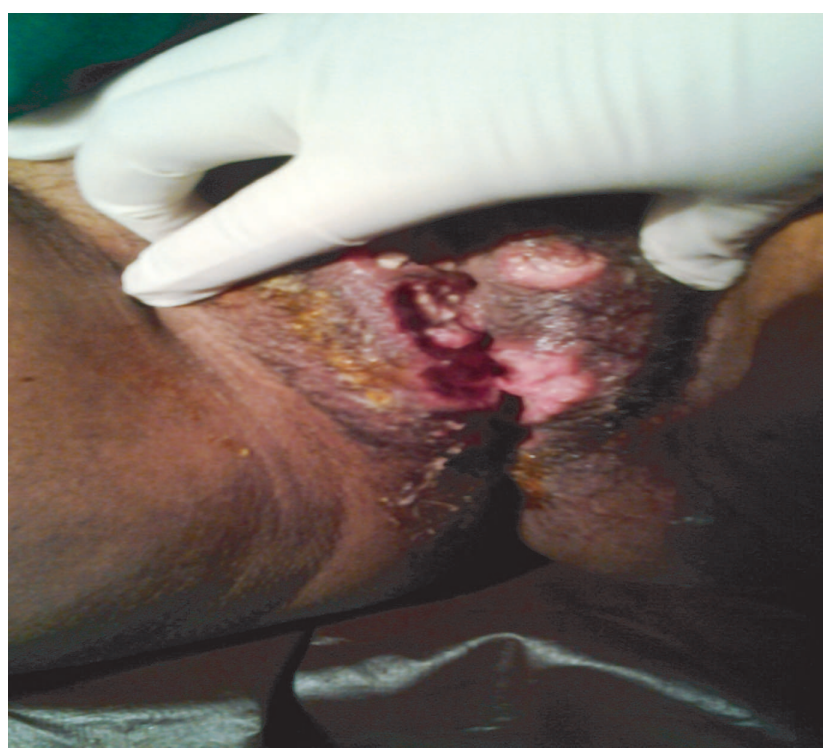

Fig 1. Extensive confluent ulceration with necrotic base on both sides of labia majora and minora

After consultation with dermatologist, we treated the patient with Inj. dexamethasone $5 \mathrm{mg} 12$ hourly for 5 days followed by oral prednisolone for 2 weeks, tablet dapsone $100 \mathrm{mg}$ once daily, tablet colchicine $0.6 \mathrm{mg} 12$ hourly, injection ceftriaxone $1 \mathrm{gm}$ twice daily (to treat secondary infection and urinary tract infection), NSAID for pain. After 3 days patient started improving significantly and after 6 days she was discharged from hospital with oral prednisolone for 2 weeks, colchicine for 2 weeks, dapsone for 20 days and cefixime for 7 days. Patient came for follow-up 7 days and 17 days after discharge. After 17 days size of ulcer was very small with minimum pain. Erythema nodosum disappeared within 7 days.

\section{Discussion}

Behcet's disease is a chronic relapsing occlusive vasculitis of unknown aetiology. It can affect all sizes and types of arteries and veins. This results in a wide variety of symptoms and complications that can affect almost any part of the body. The most common sites of involvement include the eyes, mouth, brain, skin, lungs, joints, genitals, and gastrointestinal tract. ${ }^{4}$ The commonest presentation is oral or genital ulcer, skin or eye involvement. Our patient also presented with genital ulcer and skin involvement. Oral aphthous ulcer was not present at the time of diagnosis but patient gave history of recurrent oral lesions which she had not considered as serious and did not make any consultation with any physician. According to ISGBD criteria (recurrent oral ulcer, presence of genital ulcer and erythema nodusum) patient was diagnosed as a case of Behcet's disease. ${ }^{1}$ Our patient also fulfils the ICBD criteria (genital ulcer 2 points and skin lesion - erythema nodusum 1 point; total 3 points for diagnosis). ${ }^{11}$ However, pathergy test was negative for this patient. But histopathology report supports diagnosis of Behcet's disease (angiocentric mononuclear cells and neutrophilic infiltrate with few extravasation of RBCs). Other available tests to exclude other causes of genital ulcer were negative. The age of our patient is similar to the patients' mean age at the time of diagnosis.

It is possible that a diagnosis of Behcet's disease can be missed or delayed because it is a rare disease and there is no specific diagnostic test for it, and because some symptoms can resemble symptoms of less serious conditions, such as cold sores, canker sores, genital herpes. Our patient had typical presentation for Behcet's disease according to the ISGBD criteria ${ }^{1}$, but she was initially misdiagnosed as having viral or bacterial infections and got treatment empirically for viral and bacterial infections and for syphilis also.

Generally Behcet's disease responds well to steroids. In addition to corticosteroids, immunosuppressant drugs are indicated in severe cases and when vital organs are involved. ${ }^{10}$ Our patient responded very well to the steroids (injection dexamethasone for 5 days followed by oral prednisolone) as well as immune modulators (dapsone and colchicine).

The prevalence of Behcet's disease in Bangladesh is unknown. We assume that many cases remain undiagnosed or unreported. This disease has the 
potential to affect several organs and it can lead to blindness or even death. So timely diagnosis, prompt treatment and long term follow-up are needed. In our patient, diagnosis was made early and treatment was also started early. Although at present no systemic involvement is present, long term follow-up is required. Despite the inclusive criteria set forth by the International Study Group and ICBD, there are cases where not all the criteria can be met at the same time and therefore a diagnosis cannot readily be made. There is, however, a set of clinical findings that a physician can rely upon in making a tentative diagnosis and Behcet's disease should be a differential diagnosis for a patient who presents with symptoms and signs compatible with the disease.

\section{References}

1. International Study Group for Behçet's Disease. Criteria for diagnosis of Behçet's disease. Lancet 1990; 335 (8697): 1078-1080.

2. Andreas A, Nestor P, Helmut O, Peter M, Lothar K, Christos CZ. Epidemiology and clinical manifestations of Adamantiades-Behcet disease in Germany-current pathogenetic concepts and therapeutic possibilities. Journal der Deutschen Dermatologischen Gesellschaft 2006; 4(1): 49-66.

3. Dogan SM, Birdane A, Korlemaz C, Ata N, Timuralp B. Right ventricular thrombus with Behcet's syndrome: successful repair with warfarin and immunosuppressive agents. Tex Heart Institute Journal 2007; 34(3): 360-362.
4. Arthritis Research UK. Available at http://www. arthritisresearchuk.org/arthritis-information/conditions/ behcets-syndrome.aspx. Accessed June 2015.

5. Yurdakul S, Hamuryudan V, Yazici H. Behcet syndrome. Curr Opin Rheumatol 2004; 16: 38-42.

6. de Menthon M, Lavalley MP, Maldini C, Guillevin L, Mahr A. HLA-B51/B5 and the risk of Behcet's disease: a systematic review and meta-analysis of case-control genetic association studies. Arthritis Rheum 2009; 61: 1287-1296.

7. Calamia KT, Wilson FC, NIcen M, Crowson CS, Gabriel SE, Kremers HM. Epidemiology and clinical characteristics of Behcet's disease in the US: a population-based study. Arthritis Rheum 2009; 61: 600-604.

8. Erkan F, Gul A, Tasali E. Pulmonary manifestations of Behcet's disease. Thorax 2001; 56: 572-578.

9. Kansu T, Kirkali P, Kansu E, Zileli T. Optic neuropathy in Behçet's disease. J Clin Neuroophthalmol 1989; 9(4): 277-280.

10. Hatemi G, Seyahi E, Fresko I, Hamuryudan V. Behçet's syndrome: a critical digest of the 2012-2013 literature. Clin Exp Rheumatol 2013; 31(3 Suppl 77): 108-117.

11. International Team for the Revision of the International Criteria for Behcet's Disease. Revision of the International Criteria for Behcet's Disease (ICBD). Clinical and Experimental Rheumatology 2006; 24(Suppl 42): S14-S15.

12. EULAR recommendations for the management of Behcet's disease. Ann Rheum Dis 2008; 67: 1656-1662. 